\title{
The Influence of Electromagnetic Scattering from a Permeable Sphere on the Induced Voltage across a Rotating Eccentric Coil
}

\author{
Constantinos A. Valagiannopoulos
}

Department of Radio Science and Engineering, School of Electrical Engineering, Aalto University, Espoo, Finland. Email: konstantinos.valagiannopoulos@aalto.fi

Received November $16^{\text {th }}, 2010$; revised December $20^{\text {th }}, 2010$; accepted December $27^{\text {th }}, 2010$.

\begin{abstract}
Electromagnetic scattering and electromagnetic induction are research topics not directly associated to each other. In this work, these two different concepts are combined in a model constituted by a rotating circular coil with a dielectric spherical core at a fixed eccentric position. The scope of the analysis is to examine the effect of a permeable object on the production of the alternating voltage. Methods and formulas from both scattering and induction have been utilized for the derivation of the developed potential difference around the moving loop. Several graphs of the voltage output with respect to the geometrical and material characteristics of the configuration, are presented and discussed.
\end{abstract}

Keywords: Electromagnetic Induction, Electromagnetic Scattering, Penetrable Dielectric Sphere, Rotating Loop

\section{Introduction}

The electromagnetic induction is defined as the development of voltage across a closed conductor with timevarying magnetic flux through it. The physics that govern the inductive experiments have been mathematically examined in a number of elementary treatises. The relationship between the various induction laws is summarized in [1], where Cohn advocates that the combined use of both motional and transformer induction assures the validity of the produced results. A complete report reviewing the major developments and identifying important trends in the broad field of geophysical electromagnetic induction is given in [2]. Moreover, a rudimentary study on induction inside a rotating coil surrounded by a rigid conductor of finite or infinite extent has been provided in [3]. The analysis is based on integral solutions of the field equations and leads to the conclusion that the induced magnetic fields depend on the relative symmetry of the rotator.

The electromagnetic scattering is defined as the modification of the incident field in the presence of an obstacle through the fulfillment of the boundary conditions, and numerous researches are performed on this topic. In [4], the point-source scattering by an electrically large conducting sphere has been discussed, where Bessel functions of complex order are utilized. In addition, the quasi-magnetostatic solution for a permeable prolate spheroid under arbitrary excitation by a time-harmonic primary field has been obtained by using the separation of variables method with vector spheroidal wave functions [5]. Finally in [6], a simplified solution is obtained to the problem of a radiating loop in the presence of a metallic core.

In this work, we combine the two aforementioned issues (induction and scattering) by considering a structure comprised of a rotating thin circular loop and an eccentrically positioned penetrable spherical core, illuminated by a plane wave. The rotation happens around the eccentric axis passing through the centre of the spherical cavity. The magnetic vector potential at the position of the thin closed wire is evaluated with use of spherical eigenfunction expansions and through the enforcement of the boundary conditions. The variance of magnetic flux is computed from the line integral of the electric field around the metallic coil. The DC offset and the RMS value of the produced voltage are represented in several graphs with respect to the size of the sphere, the material of the core and the excitation parameters as well. By inspection of the variations, one can reach various useful and applicable conclusions. In particular, one can choose the texture, the position and the radius of the scatterer 
that should be posed eccentrically inside the ring in order to obtain maximum output voltage. The engineer could utilize the dielectric core, when constructing an electromagnetic induction device, as an "optimizer" for regulating the produced potential.

\section{Mathematical Concept}

The configuration of the examined problem is shown in Figure 1(a) where the spherical coordinate system $(r, \theta$, $\varphi)$ and the equivalent Cartesian one $(x, y, z)$ are also defined. The origin $O$ coincides with the center of a spherical volume (region 1) of radius $a$, filled with dielectric material of relative permittivity $\varepsilon_{1}$ and relative magnetic permeability $\mu_{1}$. The scatterer is posed into vacuum (region 0$)$ with intrinsic parameters $\left(\varepsilon_{0}, \mu_{0}\right)$. A thin circular metallic loop of radius $b>a$, is shown on the $x-y$ plane, with its center $K$ at $(x, y, z)=(0, d, 0)$ with $b>d$, located eccentrically to the spherical core. The closed wire is rotated with respect to $x$ axis with circular frequency $\omega$ in the presence of an $x$-polarized plane wave $\mathbf{E}_{0, \text { inc }}$, with magnitude $Q$ (in $V / m$ ), advancing towards the negative $z$ semi-axis. Mind that the harmonic time dependence of the incident field, is of the form $\exp \left(-i \omega_{0} t\right)$; it has circular frequency $\omega_{0} \neq \omega$. In Figure 1(b), we present a side view of the device as appeared from the positive $x$ semi-axis, when the frame is rotated by angle $\omega t$, at an arbitrary time $t$.

The polar radius $R(\varphi)$ of the eccentric circular loop at $t$ $=0($ Figure 1(a)) is determined by applying the law of cosines to the shaded triangle, yielding to:

$$
R(\varphi)=d \sin \varphi+\sqrt{b^{2}-d^{2} \cos ^{2} \varphi} .
$$

It is necessary to extract the parametric equation set of the rotated coil denoted by $\{x=X(\varphi, t), y=Y(\varphi, t)$, $z=Z(\varphi, t)\}$ The azimuthal angle $\varphi \in[0,2 \pi]$ will play the role of the parameterization variable even when the object does not belong exclusively to $x-y$ plane. At this point, it is proper to make clear that there is no practical importance in considering the filamentary loop as a torus possessing non-negligible thickness. In the vast majority of works concerning voltage production by electromagnetic induction, the coil boundaries are constructed from wires with infinitesimal transversal dimensions. Furthermore, the assumed oscillation frequency is kept quite low and thus the electrical size of the wire would be extremely small even it is not filamentary. In such a case, the solution to the compound scattering problem from both the dielectric sphere and the metallic torus would lead to almost identical results to those assuming thinwire approximations.

As the closed wire is rotated with respect to $x$ axis, the corresponding coordinate $X(\varphi, t)$ will be fixed, independent from the angle $\omega t$ and equal to $P(\varphi) \cos \varphi$. The rest two equations are derived by projecting the other edge of length $P(\varphi) \sin \varphi$, positioned at angle $\omega t$, upon the axes $x$ and $z$ (see Figure 1(b)). Accordingly, one obtains the following expressions:

$$
\begin{aligned}
& X(\varphi, t)=R(\varphi) \cos \varphi \\
& Y(\varphi, t)=R(\varphi) \sin \varphi \cos \omega t \\
& Z(\varphi, t)=R(\varphi) \sin \varphi \sin \omega t
\end{aligned}
$$

The parametric representation of the curve in spherical coordinates $\{r=P(\varphi, t), \theta=\Theta(\varphi, t), \varphi=\Phi(\varphi, t)\}$ for each $\varphi \in[0,2 \pi]$, is given by:

$$
\begin{aligned}
& \mathrm{P}(\varphi, t)=R(\varphi) \\
& \Theta(\varphi, t)=\arccos (\sin \varphi \sin \omega t) \\
& \Phi(\varphi, t)=\arctan (\tan \varphi \cos \omega t)
\end{aligned}
$$

According to Faraday's law of induction [7], the induced voltage across a closed metallic wire $(W)$, is defined as the line integral of the local electric field around the loop. In case of a monochromatic electric field with circular frequency $\omega_{0}$, the related formula is given below:

$$
U=\operatorname{Re}\left[e^{-i \omega_{0} t} \int_{(W)} \mathbf{E}_{0} \cdot d \mathbf{W}\right]
$$

It should be stressed that $\mathbf{E}_{0}$ does not denote the real, time-dependent electric field into vacuum, but the corresponding complex phasor. In case the field quantities are expressed in terms of the spherical coordinate system $(r$, $\theta, \varphi)$, the Cartesian components are given by [8]:

$$
\begin{aligned}
& {\left[\begin{array}{c}
E_{0 x}(r, \theta, \varphi) \\
E_{0 y}(r, \theta, \varphi) \\
E_{0 z}(r, \theta, \varphi)
\end{array}\right] } \\
= & {\left[\begin{array}{ccc}
\cos \varphi \sin \theta & \cos \varphi \cos \theta & -\sin \varphi \\
\sin \varphi \sin \theta & \sin \varphi \cos \theta & \cos \varphi \\
\cos \theta & -\sin \theta & 0
\end{array}\right] \cdot\left[\begin{array}{l}
E_{0 r}(r, \theta, \varphi) \\
E_{0 \theta}(r, \theta, \varphi) \\
E_{0 \varphi}(r, \theta, \varphi)
\end{array}\right] }
\end{aligned}
$$

Once these functions are determined, the line integral of (4) is particularized to give [9]:

$$
U(t)=\operatorname{Re}\left[e^{-i \omega_{0} t} \int_{0}^{2 \pi}\left(e_{0 x}(\varphi, t) X_{\varphi}(\varphi, t)+e_{0 y}(\varphi, t) Y_{\varphi}(\varphi, t)+e_{0 z}(\varphi, t) Z_{\varphi}(\varphi, t)\right) d \varphi\right]
$$




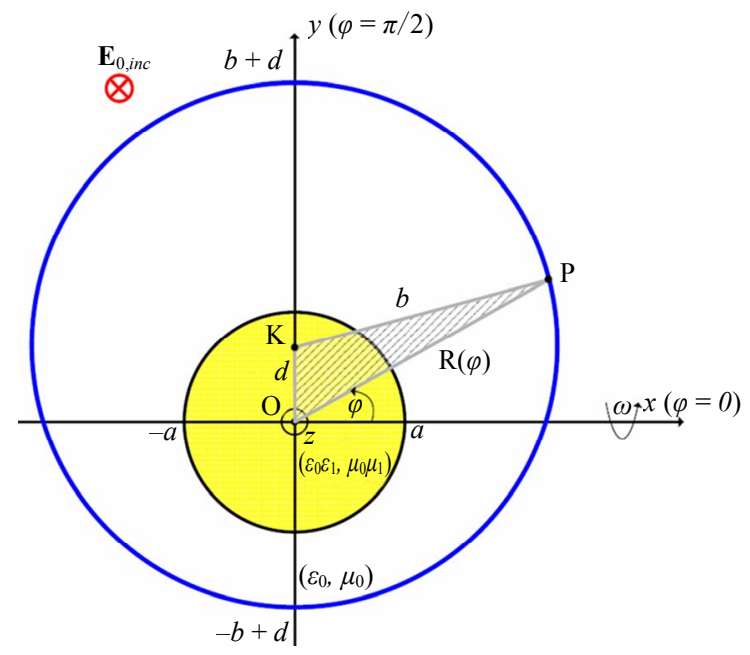

(a)

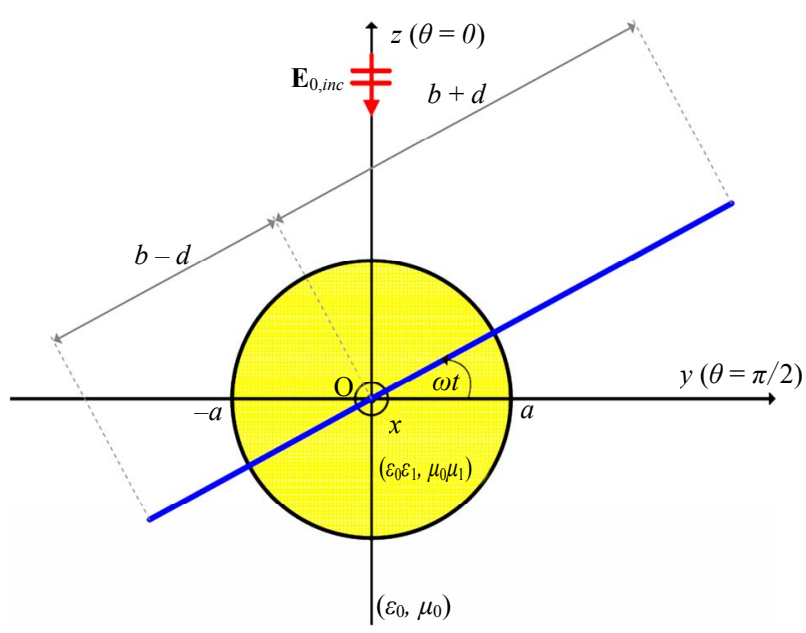

(b)

Figure 1. The physical configuration of the investigated device: (a) As viewed from the positive $z$ semi-axis. (b) As viewed from the positive $x$ semi-axis. The red arrow denotes the moving direction of the incident plane wave, not the actual vector of the electric field.

where subscript $\varphi$ corresponds to the azimuthal partial derivative of the related function. The small-e functions $\left\{e_{0 x}, e_{0 y}, e_{0 z}\right\}$ are the electric field components evaluated around the moving circular loop:

$$
\begin{aligned}
& e_{0 x}(\varphi, t)=E_{0 x}(\mathrm{P}(\varphi, t), \Theta(\varphi, t), \Phi(\varphi, t)) \\
& e_{0 y}(\varphi, t)=E_{0 y}(\mathrm{P}(\varphi, t), \Theta(\varphi, t), \Phi(\varphi, t)) \\
& e_{0 z}(\varphi, t)=E_{0 z}(\mathrm{P}(\varphi, t), \Theta(\varphi, t), \Phi(\varphi, t))
\end{aligned}
$$

Thus, the only prerequisite to use expression (6) in computing the induced voltage, is the explicit form of the total electric field into vacuum, expressed in spherical coordinates.

The electric field into vacuum background is comprised of the incident and the scattering component $\mathbf{E}_{0}=$ $\mathbf{E}_{0, \text { inc }}+\mathbf{E}_{0, \text { scat }}$, where $\mathbf{E}_{0, i n c}=\mathbf{x} Q \exp \left(-i k_{0} z\right)$ and $k_{0}=$ $\omega_{0} \sqrt{\varepsilon_{0} \mu_{0}}$. The electric field into vacuum region is computed with use of spherical eigenfunctions and the following series expansion [10]:

$$
e^{-i k_{0} r \cos \theta}=\sum_{n=1}^{+\infty}(-i)^{n+1}(2 n+1) P_{n}^{0}(\cos \theta) j_{n}\left(k_{0} r\right)
$$

The symbol $P_{n}^{m}(x)$ corresponds to the Legendre function of degree $n$, order $m$ and argument $x$. The spherical Bessel $j_{n}(x)$ and the spherical Hankel of the first kind $h_{n}(x)$ are well-known [11]. The Riccati functions are defined as $z_{n}^{d}(x)=d\left[z_{n}(x)\right] / d x$, where $z_{n}(x)$ is the spherical Bessel or Hankel function. Once the boundary conditions at $r=a$ are imposed, the respective scattering components of the electric field are given by:

$$
E_{0 r, s c a t}(r, \theta, \varphi)=-Q \cos \varphi \sum_{n=1}^{+\infty} S(n) P_{n}^{1}(\cos \theta) \frac{h_{n}\left(k_{0} r\right)}{k_{0} r}
$$

where:

$$
\begin{gathered}
S(n)=(-i)^{n+1}(2 n+1) \frac{j_{n}^{d}\left(k_{1} a\right) j_{n}\left(k_{0} a\right)-\varepsilon_{1} j_{n}^{d}\left(k_{0} a\right) j_{n}\left(k_{1} a\right)}{j_{n}^{d}\left(k_{1} a\right) h_{n}\left(k_{0} a\right)-\varepsilon_{1} h_{n}^{d}\left(k_{0} a\right) j_{n}\left(k_{1} a\right)} \\
T(n)=i^{n}(2 n+1) \frac{j_{n}^{d}\left(k_{1} a\right) j_{n}\left(k_{0} a\right)-\mu_{1} j_{n}^{d}\left(k_{0} a\right) j_{n}\left(k_{1} a\right)}{j_{n}^{d}\left(k_{1} a\right) h_{n}\left(k_{0} a\right)-\mu_{1} h_{n}^{d}\left(k_{0} a\right) j_{n}\left(k_{1} a\right)}
\end{gathered}
$$


The wavenumber in region 1 is defined by $k_{1} \omega_{0} \sqrt{\varepsilon_{1} \mu_{1}}$.

As one can notice, the mathematical formulation above is divided in two subsections; the first one concerns the electromagnetic induction and the second one examines the electromagnetic scattering. The induced voltage around the loop (first subsection) is solely dependent on the electromagnetic field and the shape/position of the coil. Successful manipulation of the boundary value scattering problem (second subsection) gives the required electromagnetic field in explicit form. Accordingly, there are two successive series of algebraic operations which help us understanding the combined induction/scattering consideration. In this sense, the two concepts are not treated independently each other, because the solution to the scattering problem is a prerequisite to solving the induction one. That is why the dielectric core cannot be present in our configuration, in case one ignores the scattering procedure. In particular, the only mechanism through which the permeable sphere participates in the investigated context is the modification of the electromagnetic field externally to it.

It is also known [7] that the developed voltage around a loop with area (S) is proportional to the time rate of change of the magnetic flux through it, namely:

$$
U=-\operatorname{Re}\left[\frac{d}{d t}\left(e^{-i \omega_{0} t} \int_{(S)} \mathbf{B}_{0} \cdot d \mathbf{S}\right)\right]
$$

where $\mathbf{B}_{0}$ is the magnetic field into vacuum. The area integral above will be used alternatively for the evaluation of the induced potential difference in order to validate our results.

\section{Numerical Results}

Prior to proceeding to the numerical results, we should determine the intervals into which the input parameters belong. The radius of the metallic loop can take values within the range $0.5 \mathrm{~m}<b<2.5 \mathrm{~m}$ (in most cases equal to $b=1 \mathrm{~m}$ ). The frequency of the excitation wave is moderate (usually equal to $\omega_{0}=200 \pi \mathrm{rad} / \mathrm{sec}$ ), being taken between the limits: $2 \pi \mathrm{rad} / \mathrm{sec}<\omega_{0}<400 \pi \mathrm{rad} / \mathrm{sec}$. When it comes to the spherical scatterer, its relative permittivity does not affect substantially the results and thus is kept constant throughout the numerical simulations $\left(\varepsilon_{1}\right.$ $=5$ ). The relative magnetic permeability of the sphere possesses usual magnitudes, that is $1<\mu_{1}<2.5$ and normally is assigned the value $\mu_{1}=2$. To the query "why does the permeability vary?", one shall respond that the characteristics of the sphere's material are treated as degrees of freedom in designing the device and therefore can vary. As far as the values of $\mu_{1}$ are concerned, they are chosen close to those owned by common composite materials [12]. Instead of the radius of the sphere, we use the normalized parameter $a /(b-d) \in[0,1]$ as the core should be kept internal to the rotating ring. The eccen- tricity ratio $d /(b-d) \in[0,1]$ is also utilized to quantify the relative transposition of the scatterer. In the following graphs, two quantities are mainly represented; the DC offset and the RMS value of the induced voltage, defined below:

$$
\begin{aligned}
U_{d c} & =\frac{\omega}{2 \pi} \int_{0}^{\frac{2 \pi}{\omega}} U(t) d t \\
U_{r m s} & =\sqrt{\frac{\omega}{2 \pi} \int_{0}^{\frac{2 \pi}{\omega}}\left[U(t)-U_{d c}\right]^{2} d t}
\end{aligned}
$$

The amplitude of the plane wave $Q$ is a trivial parameter and therefore is chosen high enough to give realistic values for the output voltages.

In Figure 2(a), the RMS component of the produced voltage is shown as function of the normalized sphere

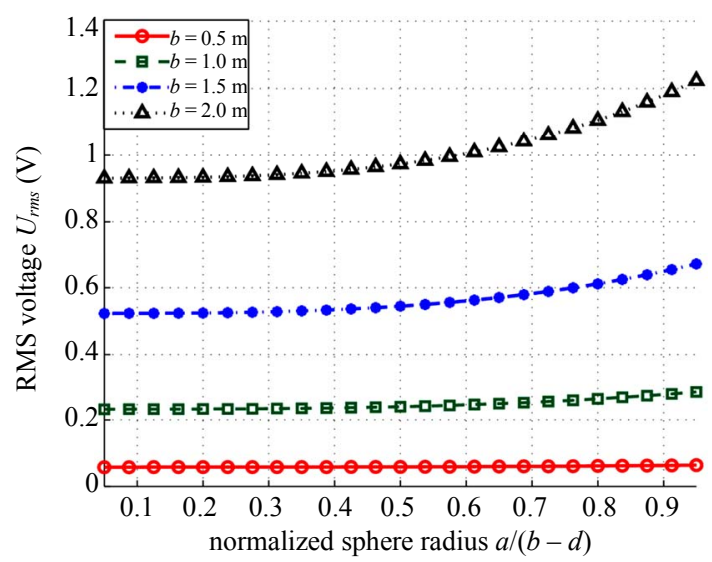

(a)

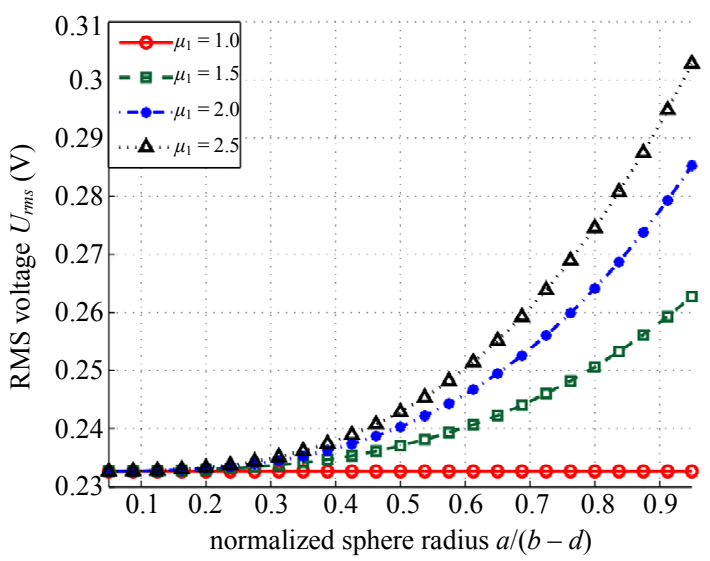

(b)

Figure 2. The RMS induced voltage $U_{r m s}$ as function of the normalized radius of the spherical scatterer $a /(b-d)$, (a) for various sizes of the loop $b\left(\mu_{1}=2\right)$, (b) for various magnetic permeabilities of the core $\mu_{1}(b=1 \mathrm{~m})$. Plot parameters: $d=$ $0.2 \mathrm{~m}, \varepsilon_{1}=5, \omega=200 \pi \mathrm{rad} / \mathrm{sec}, \omega_{0}=200 \pi \mathrm{rad} / \mathrm{sec}, Q=10^{5}$ $\mathrm{V} / \mathrm{m}$. 
radius for constant eccentric position $d$ corresponding to several sizes of the rotating loop. The maximum magnetic flux through the circular wire (and implicitly the induced voltage) is proportional to the size of this ring. Note also that the normalized radius of the sphere plays rather unimportant role when it is kept low. On the contrary, when the scatterer gets close to the frame, there is an amplifying effect on the measured quantity, which gets more significant for larger loops. This is a natural result because for fixed $b, d$, the available manoeuvring area for the sphere to move gets restricted when the of the loop is small. In Figure 2(b), the RMS value of the produced voltage is represented with respect to the same variable $a /(b-d)$ for various magnetic permeabilities of the sphere. One could also notice the exponentially increasing behavior of the curves which remarks the beneficial influence of the scatterer's radius on the induced voltage. In addition, this upward sloping trend is proportional to the magnetic permeability of the spherical core, while there is no variation when the obstacle is magnetically inert. In other words, the size and the magnetic density of the dielectric core provide the design engineer with two additional degrees of freedom in constructing an efficient voltage generator.

In Figure 3, we show the measured response with respect to the permeability of the core $\mu_{1}$, for several eccentricity ratios $d /(b-d)$. It should be stressed that the eccentricity ratio affects crucially the RMS value of the induced voltage which means that the degree of asymmetry reinforces the recorded quantity. In this way, the permeable sphere could play the role of a tuner with which the magnitude of the output is chosen at will by changing the eccentric position of the sphere. Also, all the curves coincide at $\mu_{1}=1$ and then increase gradually for magnetically denser construction materials. It seems that when $\mu_{1}=1$, the dielectric core becomes completely transparent to the incident electromagnetic field regardless of its position.

In Figure 4(a), the RMS component of the produced voltage is shown in a contour plot with respect to the rotation frequency of the loop and the oscillation frequency of the incident plane wave. For increasing $\omega_{0}$, the recorded quantity gets reinforced with a pace negatively related to $\omega$. Once the rotation frequency gets larger, there is either a stability in the measured output (modest $\omega_{0}$ ) or a magnitude boost (substantial $\omega_{0}$ ). It should be also remarked that when $\omega$ is very low, rapid variations in $U_{r m s}$ are observed for little change of $\omega_{0}$. This chaotic behavior is attributed to the fact that, in case $\omega \rightarrow 0$, the magnetic flux is considerably affected even by the slightest variance in the frequency of the alternating field. In Figure 4(b), the DC offset $U_{d c}$ is represented for the

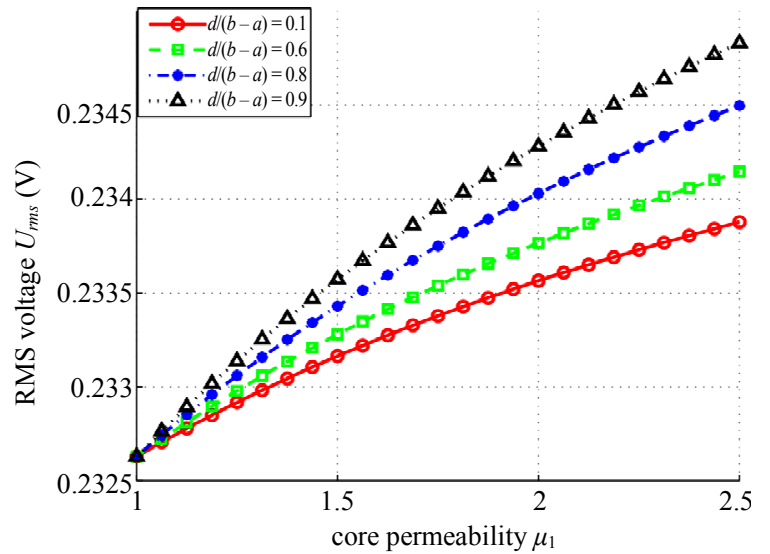

Figure 3. The RMS induced voltage $U_{r m s}$ as function of the core magnetic permeability $\mu_{1}$, for several eccentricity ratios $d /(b-a)$. Plot parameters: $a=0.2 \mathrm{~m}, b=1 \mathrm{~m}, \varepsilon_{1}=5, \omega=$ $200 \pi \mathrm{rad} / \mathrm{sec}, \omega_{0}=200 \pi \mathrm{rad} / \mathrm{sec}, Q=10^{5} \mathrm{~V} / \mathrm{m}$.

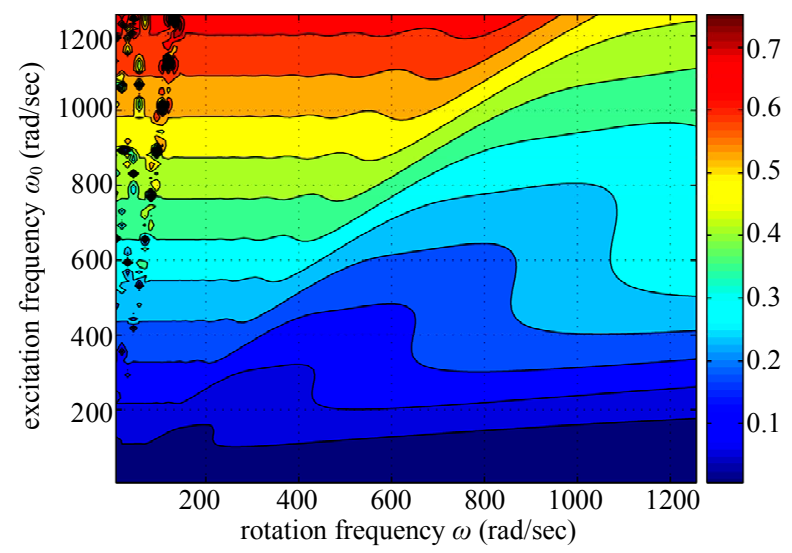

(a)

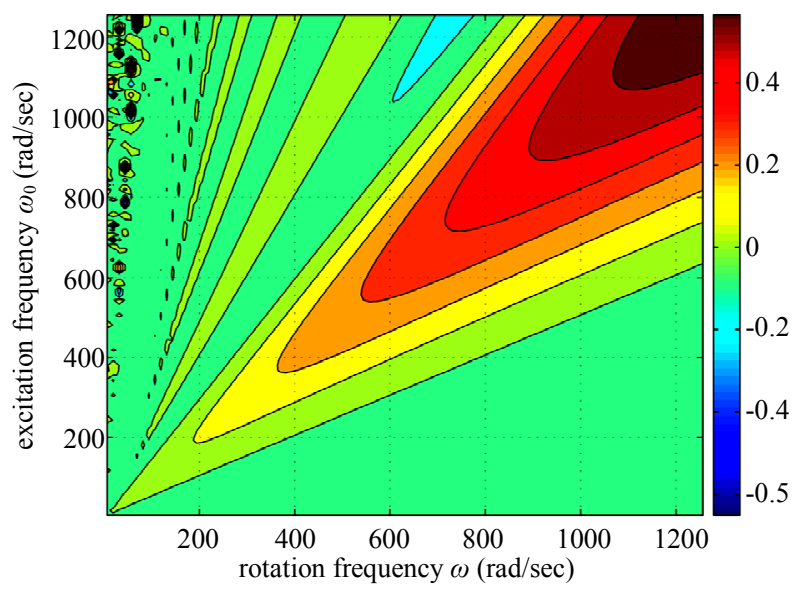

(b)

Figure 4. Contour plots of (a) the RMS induced voltage $\boldsymbol{U}_{r m \mathrm{~s}}$ and (b) the DC component of the produced voltage $U_{d c}$, with respect to the rotation frequency $\omega$ and the excitation frequency $\omega_{0}$. Plot parameters: $a=1 / 3 \mathrm{~m}, d=1 / 3 \mathrm{~m}, b=1 \mathrm{~m}$, $\varepsilon_{1}=5, \mu_{1}=2, Q=10^{5} \mathrm{~V} / \mathrm{m}$. 
same set of parameters. Note that in all the previous examples, the presence of the scatterer makes the produced oscillating voltage to have nonzero average value owning similar waveforms to $U_{r m s}$. This is not the case; when $\omega$ is chosen close to $\omega_{0}$, there is a substantial increase for growing frequencies. After numerical trials, we concluded that the shape of the equal $U_{d c}$ levels in Figure 4(b) resemble rotated hyperbolas with narrow extent following an asymptotic law of:

$$
U_{d c}=O\left[5 \omega \omega_{0}-\frac{3}{2} \omega^{2}-3 \omega_{0}^{2}\right]
$$

In Figure 5, we show in contour plot the relative difference between the estimation of the RMS voltage via normal expression (4) and its evaluation through the alternative Formula (11). This percent error is represented as function of the quantities $(a / b, d / b)$, where $a+d \leq b$, and one can observe that its magnitude is very low (below $0.0002 \%$ ). As the truncation limit (number of terms summed) in the series (9) is kept fixed, the error gets more significant for more sizeable scatterers. Additionally, the more eccentric is the position of the sphere, the more substantial is the recorded quantity. As far as most of the numerical results represented in previous figures are concerned, they have been verified through the area integral (11) and remarkable coincidence with (4) is exhibited.

\section{Conclusions}

In this work, we examine the induction of electromagnetic voltage across a rotating circular loop, in the presence of an eccentric dielectric sphere under a low-frequency, plane-wave excitation. Similar topics combining two

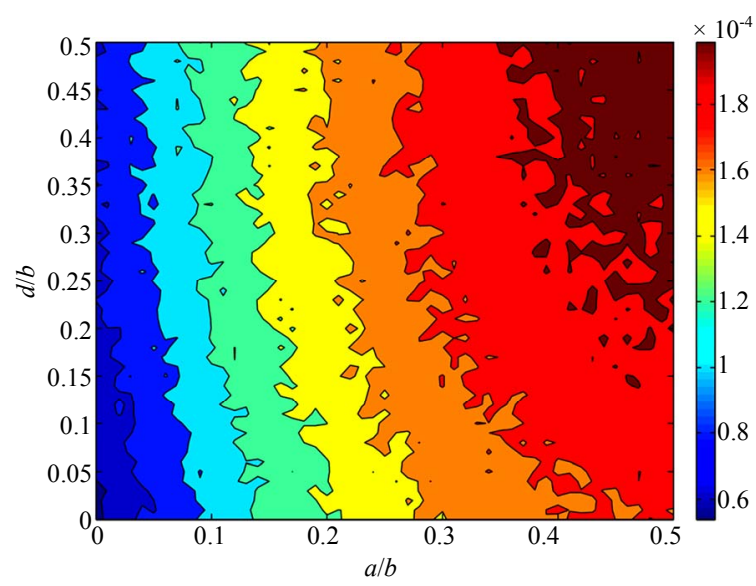

Figure 5. Contour plot of the percent error in computing the RMS induced voltage $U_{r m s}$ through (4) and (11), with respect to the size of the scatterer $a / b$ and its eccentric position $d / b$. Plot parameters: $b=1 \mathrm{~m}, \varepsilon_{1}=5, \mu_{1}=2, Q=10^{5}$ $\mathbf{V} / \mathbf{m}$. fundamental phenomena in electromagnetics (induction and scattering) have not been studied yet.

The variation of the measured output is represented as function of the sphere's characteristic parameters and several conclusions are drawn describing its effect on the magnetic flux through the coil. An interesting expansion of the present study would be to assume a spherical scatterer with inhomogeneities and/or anisotropies, or to modify slightly the shape of the core using oblate spheroid coordinates and functions. Also, closed wires of arbitrary curvature rotating around arbitrary axes could be also investigated with use of the same techniques.

\section{REFERENCES}

[1] G. I. Cohn, "Electromagnetic Induction," Electrical Engineering, Vol. 68, No. 5, May 1949, pp. 441-447.

[2] A. D. Chave and J. R. Booker, "Electromagnetic Induction Studies," Reviews of Geophysics, Vol. 25, No. 5, June 1987, pp. 989-1003. doi:10.1029/RG025i005p00989

[3] A. Herzenberg and F. J. Lowes, "Electromagnetic Induction in Rotating Conductors," Philosophical Transactions of the Royal Society of London, Series A, Mathematical and Physical Sciences, Vol. 249, No. 5, 1957, pp. 507-584. doi:10.1098/rsta.1957.0006

[4] C. A. Valagiannopoulos, "An Overview of the Watson Transformation Presented through a Simple Example," Progress in Electromagnetics Research, Vol. 75, 2007, pp. 137-152. doi:10.2528/PIER07052502

[5] C. O. Ao, H. Braunisch, K. O'Neill and J. A. Kong, "Quasi-Magnetostatic Solution for a Conducting and PerMeable Spheroid with Arbitrary Excitation," IEEE Transactions on Geoscience and Remote Sensing, Vol. 40, No. 4, 2002, pp. 887-897. doi:10.1109/TGRS.2002.1006370

[6] C. A. Valagiannopoulos, "Single-Series Solution to the Radiation of Loop Antenna in the Presence of a Conducting Sphere," Progress in Electromagnetics Research, Vol. 71, 2007, pp. 277-294. doi:10.2528/PIER07030803

[7] M. Sadiku, "Elements of Electromagnetics," Oxford Series in Electrical and Computer Engineering, 2001, p. 372.

[8] C. A. Balanis, "Advanced Engineering Electromagnetics," John Wiley \& Sons, New York, 1989, p. 924.

[9] R. C. Wrede and M. R. Spiegel, "Advanced Calculus," Schaumm's Outline Series, 2002, pp. 229-232.

[10] V. A. Erma, "Exact Solution for the Scattering of ElectroMagnetic Waves from Conductors of Arbitrary Shape. II. General Case," Physical Review, 1968, pp. 1544-1553. doi:10.1103/PhysRev.176.1544

[11] M. Abramowitz and I. A. Stegun, "Handbook of Mathematical Functions," National Bureau of Standards, 1970, pp. 437-438.

[12] C. A. Valagiannopoulos, "On Smoothening the Singular Field Developed in the Vicinity of Metallic Edges," International Journal of Applied Electromagnetics and Mechanics, Vol. 31, No. 3, 2009, pp. 67-77. 